\title{
The protective effect of previous COVID-19 infection in a high-prevalence hospital setting
}

\author{
Authors: Favian Narrainen, ${ }^{A}$ Madeleine Shakeshaft, ${ }^{B}$ Hibo Asad, ${ }^{C}$ Abigail Holborow, ${ }^{D}$ Ian Blyth ${ }^{\mathrm{E}}$ and \\ Brendan Healy ${ }^{\mathrm{E}}$
}

\section{Objective}

To assess the protective effect of previous COVID-19 infection for healthcare workers in a high-prevalence setting.

\section{Method}

The COVID-19 antibody and PCR results of 538 healthcare workers on wards with COVID-19 outbreaks from 1 March 2020 to 31 July 2020 were evaluated. Infection rates of the 'previously infected' and 'no evidence of previous infection' groups were compared during second-wave outbreaks between 29 September 2020 and 20 November 2020.

Results

One out of 115 individuals previously infected developed infection compared with 104 out of 423 individuals with no evidence of previous infection. Attack rates in staff previously infected was reduced significantly from $24.59 \%$ to $0.87 \%$ (odds ratio $0.027,95 \% \mathrm{CI} 0.004-0.195, \mathrm{p}<0.001$ ) when compared to the 'no evidence of previous infection' group with the same exposure risk.

Conclusion

Prior SARS-CoV-2 infection offers significant protection against reinfection and this protection lasts 4 months for the majority of individuals.

KEYWORDS: COVID-19, SARS-CoV-2, reinfection, health personnel, antibodies

DOI: $10.7861 /$ clinmed.2021-0225

\section{Introduction}

The SARS-CoV-2 pandemic has impacted significantly on communities, health services and the economy. As of 16 August

Authors: ${ }^{\text {A }}$ medical student, Swansea University, Swansea, UK; ${ }^{B}$ specialist registrar in infectious diseases and microbiology, Public Health Wales Microbiology, Singleton Hospital, Swansea, UK; C healthcare epidemiologist, Healthcare Epidemiology, Public Health Wales Microbiology, Singleton Hospital, Swansea, UK; D physician associate in microbiology and infectious diseases, Public Health Wales Microbiology, Singleton Hospital, Swansea, UK; ${ }^{\mathrm{E}}$ Consultant in microbiology and infectious diseases, Public Health Wales Microbiology, Singleton Hospital, Swansea, UK
2021 there have been 207,173,086 confirmed cases of COVID-19, including 4,361,996 deaths around the world. ${ }^{1}$ High rates of transmission have been evident across the globe and measures such as social distancing and face coverings designed to reduce transmission have been unable to completely prevent propagation of infection in the community. Enhanced measures in healthcare settings, including use of personal protective equipment (PPE), have also been unable to prevent spread in the hospital environment. $^{2}$

Vaccination is now being rolled out in the UK in an effort to reduce the impact of COVID-19 on society. Data from vaccination studies demonstrate protection for up to 2 months. ${ }^{3}$ Further data on duration of protection afforded by the vaccine will be available in due course. In the interim, it is possible to gain some information on the duration of protection beyond 2 months by analysing data from natural infection acquired during the first wave in high-prevalence settings.

Immunity post SARS-CoV-2 infection is not absolute. Reinfection has been confirmed in a number of reports ${ }^{4-9}$ (including confirmation by whole-genome sequencing (WGS) in some cases), ${ }^{5-7}$ demonstrating that previous infection is not $100 \%$ protective even when SARS-CoV-2 antibodies develop..$^{8,9}$ However, the level of protection afforded by previous infection and how likely someone is to develop a second infection is still unclear.

Understanding the extent of post-infection immunity on preventing reinfection will have important implications for public policy, guiding behaviour and infection control in healthcare settings and beyond.

Here we present a retrospective cohort data analysis that investigated the impact of previous infection including baseline antibody on reinfection in healthcare workers (HCWs) during periods of high prevalence of SARS-CoV-2 at a university health board in South Wales, UK, during the second wave.

\section{Methods}

Background

From March 2020 onwards, symptomatic HCWs were tested for SARS-CoV-2 using PCR testing of combined nasal and oropharyngeal or oropharyngeal swabs (according to the assay used). Criteria for testing changed throughout the pandemic in line with evolving evidence and overall capacity for testing. Screening for infection occurred on a number of occasions in outbreak areas when it became apparent that transmission during 
asymptomatic infection was common. Testing capacity limited screening on some occasions.

A SARS-CoV-2 antibody assay testing programme was conducted in the health board during the period from 2 June 2020 to 7 July 2020. Healthcare workers working on all sites were invited to have antibody testing carried out. Testing was voluntary but uptake was high. A total of 7,963 antibody tests were carried out in this period, $64 \%$ of the total workforce $(12,500$ employees). No other antibody serology data were available past this point.

Lockdown in South Wales began on 23 March 2020 and ended on 1 June 2020. Following the lockdown, infection rates in the hospital and the community were very low. Infection rates began to increase significantly again towards the end of September 2020.

Between 29 September 2020 and 20 November 2020,

prevalence in the hospital increased and ward areas experienced outbreaks of infection. All symptomatic HCWs were able to access testing. Asymptomatic testing was also carried out on a number of outbreak areas. Rates of infection between the two cohorts ('previously infected' and 'no evidence of previous infection') during this second wave were compared.

\section{Cohort}

Individuals were included if they worked on a ward during a period of high prevalence (an outbreak ward) and had close clinical contact with patients. These wards were defined as six medical wards, one surgical ward and one rehabilitation ward. No COVID-19 cohort wards were included. Individuals (nurses and healthcare support workers) were identified from the working roster for the outbreak period (from the first patient case to the last patient case). As such, included individuals had a similar exposure risk. PPE use was the same on all wards.

\section{Definition of groups}

Individuals were categorised according to infection status following the first wave of infections in the area (1 March 2020 to 31 July 2020) as:

> 'Evidence of previous infection' - a positive PCR result or a positive antibody test or

$>$ 'No evidence of previous infection' - a negative antibody test and no evidence of a previous positive PCR result.
Individuals were further categorised according to infection status after a period of high prevalence in their ward during the second wave (29 September 2020 to 20 November 2020) as:

$>$ 'Infected in the second wave' - a positive PCR result or

> 'Not infected in the second wave' - a negative PCR result or no PCR test carried out.

Reinfection was defined as infection during the second wave in the 'evidence of previous infection' group.

\section{Asymptomatic screening}

Asymptomatic screening was carried out on a ward if the outbreak management team deemed staff screening was appropriate based on the epidemiology and prevalence of infection of the ward at the time. Criteria included new hospitalacquired infection in a ward area without an identified exposure event or widespread, uncontrolled transmission over a ward area. Screening of asymptomatic staff typically occurred at a one-off time point as soon after unexpected or widespread transmission had been identified, as testing capacity allowed. Details of asymptomatic screening by ward area are shown in Table 1. There were many reasons why staff were not screened, including declined screening, did not turn up for/insufficient time to present for screening, not working during the screening period, agency staff, or logistics. Most reasons would have affected both groups equally, although it is possible that individuals who had been previously infected would have been more likely to decline screening. The commonest reason for not being screened was not working during the risk period just prior to screening, although they would have worked on the ward during the outbreak period subsequently.

The outbreak periods were identified by infection control teams based on prevalence of infection amongst patients and staff on the ward, and retrospectively defined according to the first and last recognised cases.

\section{Laboratory assays (PCR and antibodies)}

PCR testing was performed on a number of platforms according to availability, capacity and urgency of the test result. Assays used include an in-house assay (E gene), the Cepheid GeneXpert

Table 1. Number of healthcare workers with and without evidence of previous infection swabbed during the second wave of SARS-CoV-2 outbreak

$\begin{array}{lllll}\text { Ward } & \text { Total exposed staff } & \text { Number screened } & \begin{array}{l}\text { Evidence of previous } \\ \text { infection - swab taken } \\ \text { at time of outbreak }\end{array} & \begin{array}{l}\text { No evidence of previous } \\ \text { infection - swab taken } \\ \text { at time of outbreak }\end{array} \\ 1 & 110 & 33 & 3 / 17(17.6 \%) & 30 / 93(32.3 \%) \\ 2 & 83 & 25 & 3 / 16(18.8 \%) & 22 / 67(32.8 \%) \\ 3 & 50 & 43 & 13 / 20(65.0 \%) & 30 / 30(100 \%) \\ 4 & 46 & 27 & 2 / 18(11.1 \%) & 25 / 28(89.3 \%) \\ 5 & 69 & 69 & 14 / 14(100 \%) & 55 / 55(100 \%) \\ 6 & 110 & 40 & 1 / 15(6.7 \%) & 39 / 95(41.1 \%) \\ 7 & 70 & 70 & 15 / 15(100 \%) & 55 / 55(100 \%) \\ \text { Total } & 538 & 307 & 51 / 115(44.3 \%) & 256 / 423(60.5 \%)\end{array}$


(N2 gene and E gene), Luminex Aries (ORF1ab gene and N gene), Genmark Eplex (N gene), Seegene Startlet (E gene, RdRP gene and $\mathrm{N}$ gene), Roche (ORF1ab gene and E gene), Perkin Elmer (ORF1ab gene and $\mathrm{N}$ gene) and the Bosphore (ORF1ab gene and E gene). In the early phase of the pandemic, the majority of samples were processed on the in-house assay. Later on, the majority of samples were processed on the Seegene.

Serum anti-SARS-CoV-2 antibody was detected using either the EUROIMMUN Anti-SARS-CoV-2 ELISA assay or the Roche Elecsys Anti-SARS-CoV-2. The majority of tests were carried out on the Roche assay. This uses a recombinant protein representing the nucleocapsid $(\mathrm{N})$ antigen in a double-antigen sandwich assay format. It preferentially detects high-affinity antibodies against SARS-CoV-2. Elecsys Anti-SARS-CoV-2 detects antibody titres, which have been shown to positively correlate with neutralizing antibodies in neutralization assays. ${ }^{10}$ The assay does not differentiate between IgG, IgM or IgA.

Results are categorised as reactive or non-reactive by a cut-off index based on the measurement of negative and positive control results. A cut-off index $\geq 1.0$ is considered reactive or positive for anti-SARS-CoV-2 antibodies. The magnitude of the measured result above the cut-off is not indicative of the total amount of antibody present in the sample. ${ }^{11,12}$

\section{Data storage and analysis}

All data were managed and stored on a secure Public Health Wales server. Data cleaning and analyses were carried out in Excel. The work that analysed routinely collected data collected as part of the COVID-19 pandemic and under the Public Health Wales establishment order was reviewed by the health board's Joint Study Review Committee (JSRC) and was deemed exempt from requiring an $\mathrm{NHS}$ ethics review. All results were analysed on password protected, encrypted NHS or Public Health Wales computers and has been written up in anonymised form to comply with information governance guidelines.

\section{Statistical analysis}

HCWs were classified into two groups according to their previous infection status as defined above.

Infection rates during the period of high prevalence were compared between the two groups based on the definitions of infection during the second wave. Attack rates of SARS-CoV-2 on $\mathrm{HCW}$ in the two groups were calculated and compared. Statistical analysis was conducted to determine the odds ratio, confidence intervals and $p$-value for the data. The odds ratio was calculated using www.medcalc.org/calc/odds_ratio.php. ${ }^{13}$ The $\mathrm{p}$-value was calculated according to Sheskin (p. 542). ${ }^{14}$ A standard normal deviate (z-value) is calculated as $\ln (\mathrm{OR}) / \mathrm{SE}\{\ln (\mathrm{OR})\}$, and the $p$-value is the area of the normal distribution that falls outside $\pm z$. $^{14}$

\section{Ethical approval}

The above data analysis project was reviewed at the local health board's Joint Study Review Committee (JSRC). The committee agreed that in accordance to the health board's processes, the project is deemed to have been exempt from requiring NHS ethics review. It was noted that the project involved NHS staff and was a retrospective review of routine data collected by the direct care team, fully anonymised at the point of analysis.

\section{Results}

Results from 538 HCWs were analysed. 115/538 (21.4\%) HCWs had evidence of previous infection (antibody and/or PCR) and 423/538 (78.6\%) HCWs had no evidence of previous infection.

99/538 (18.4\%) HCWs had a previous PCR test, of which 31/99

(31.3\%) HCWs had a previously positive PCR test. Moreover,

276/538 (51.3\%) HCWs had a previous antibody test, of which 107/276 (38.8\%) HCWs had a positive antibody test.

From the $115 \mathrm{HCWs}$ with evidence of previous infection, 84/115 (73.0\%) HCWs were previously positive by antibody only (no PCR result), 8/115 (7.0\%) HCWs were previously positive by PCR only (no antibody result) and 23/115 (20.0\%) HCWs were previously positive by PCR and antibody. Finally, 31/105 (29.5\%) who tested positive during the second wave did not have either an antibody test or a previous PCR test.

Median age of the cohort was 40 years, $12 \%$ male and $88 \%$ female. Median follow-up was 131 days (99 to 168 days).

Results of PCR testing during the period of high prevalence: 104 HCWs from the 'no evidence of previous infection' group tested PCR positive compared to one HCW from the 'evidence of previous infection' group. The attack rate was $0.87 \%$ in the 'evidence of previous infection' group compared to $24.59 \%$ in the 'no evidence of previous infection' group (odds ratio $0.027,95 \%$ CI $0.004-$ $0.195, p<0.001$ ) (Table 2). Asymptomatic screening in the second wave was carried out on $307 / 538$ (57.1\%) HCWs. 51/115 (44.3\%) HCWs from 'evidence of previous infection' group and 256/423 $(60.5 \%)$ from the 'no evidence of previous infection' group were screened.

The single case of reinfection occurred in a symptomatic individual who tested positive by PCR at the beginning of April 2020 (Roche assay, ORF1/a not detected. E gene CT value 37), by antibody serology in June (level 146 on Roche Elecsys Anti-SARS-CoV-2 assay) and again by PCR (Aries, ORF1/a CT value 23. N gene CT value 25) in late October, tested because of presence of new symptoms.

\section{Discussion}

Here we present data that shows previous infection with SARSCoV-2 provides significant protection to reinfection in HCWs that are working on hospital wards with a high prevalence of SARSCoV-2. Attack rates in staff with evidence of previous infection was reduced significantly from $24.59 \%$ to $0.87 \%$ (odds ratio 0.027 , $95 \%$ CI $0.004-0.195, p<0.001)$ when compared to a group with the same exposure risk but no evidence of previous infection.

Only one case of reinfection was detected among 115 individuals compared to 104 cases in 423 individuals without any evidence of previous infection. This data suggests that immunity developed during prior SARS-CoV-2 infection is protective for a median of 131 days. This would be in line with data from other recent publications from the USA (10-fold protection), ${ }^{15}$ Qatar (reinfection risk estimated at $0.02 \%$ and reinfection incidence rate at 0.36 per 10,000 person-weeks) and the UK, ${ }^{16,17}$ with large data sets spanning a several-month period. The recent Oxford, UK, study demonstrated that antibody responses offered protection from reinfection for most people in the 6 months following infection. ${ }^{17}$ No symptomatic reinfections were detected but one antibody-positive individual with no history of symptomatic COVID infection who subsequently developed COVID-19 and three asymptomatic individuals who tested positive by PCR following a previous infection with COVID-19 were reported. ${ }^{17}$ 
Table 2. SARS-CoV-2 attack rate of healthcare workers with and without evidence of previous infection during periods of high SARS-CoV-2 prevalence

$\begin{array}{llllllll}\text { Ward } & \begin{array}{l}\text { Total } \\ \text { exposed } \\ \text { staff }\end{array} & \begin{array}{l}\text { Evidence } \\ \text { of previous } \\ \text { infection }\end{array} & \begin{array}{l}\text { No evidence } \\ \text { of previous } \\ \text { infection }\end{array} & \begin{array}{l}\text { Evidence } \\ \text { of previous } \\ \text { infection that } \\ \text { tested positive }\end{array} & \begin{array}{l}\text { No evidence } \\ \text { of previous } \\ \text { infection that } \\ \text { tested positive }\end{array} & \begin{array}{l}\text { Attack } \\ \text { rate - evidence } \\ \text { of previous } \\ \text { infection (\%) }\end{array} & \begin{array}{l}\text { Attack rate - } \\ \text { no evidence } \\ \text { of previous } \\ \text { infection (\%) }\end{array} \\ 1 & 110 & 17 & 93 & 0 & 11 & 0.0 & 11.8 \\ 2 & 83 & 16 & 67 & 0 & 9 & 0.0 & 13.4 \\ 3 & 50 & 20 & 30 & 0 & 19 & 0.0 & 63.3 \\ 4 & 46 & 18 & 28 & 0 & 6 & 0.0 & 21.4 \\ 5 & 69 & 14 & 55 & 0 & 20 & 0.0 & 36.4 \\ 6 & 110 & 15 & 95 & 0 & 25 & 0.0 & 26.3 \\ 7 & 70 & 15 & 55 & 1 & 14 & 6.7 & 25.5\end{array}$

The interpretation of these results is not clear from the report. ${ }^{17}$ The Oxford study likely overestimates the protective efficacy of previous infection and the true figure is likely to lie somewhere between these results and those presented here. Our data complements the results from the Oxford study as it looks in detail in areas of high prevalence and thereby increases the probability of detecting reinfections. The infection rate in the Qatar study is also lower than presented here. However, the incident rate in that study in the general population is not stated and as such, the relative rate of reinfection cannot be calculated. The lower incident rate is likely a reflection of the overall lower risk of exposure/infection in that population.

There are a number of potential limitations to this report. Although immune-driven protection is the most likely explanation for the difference demonstrated, it is not possible to rule out other reasons for reduced rates of reinfection in the previously infected group such as changes in behaviour and improved compliance with measures that decrease the risk of infection (use of PPE, social distancing, etc). However, we have no data to suggest that compliance with these measures is any higher in these individuals. We did not have access to ethnicity data in our cohort, although there has been no published evidence linking ethnicity with higher rates of reinfection.

It is also possible that a small proportion of the staff with evidence of previous infection were identified in this group as a result of false-positive PCR results. This number could potentially reduce the size of the observed effect. However, the one case of reinfection was in an individual highly likely to have had true infection. This is based on positive SARS-CoV-2 PCR at the beginning of April 2020 (Roche assay, ORF1/a not detected. E gene CT value 37), antibody serology in June (level 146 on Roche Elecsys Anti-SARS-CoV-2 assay), and subsequent true reinfection based on new symptoms and strongly positive SARS-CoV-2 PCR in late October (Aries, ORF1/a CT value 23. N gene CT value 25). The impact of any such effect is therefore likely to be negligible. It is important to note that sequencing results were not available because the CT value on the initial positive swab was too high to enable WGS to take place (WGS is carried out on samples with CT <30).

Similarly, a small proportion of staff without evidence of previous infection may have been infected previously because some staff did not have either an antibody test or a previous PCR test and both PCR and antibody can miss genuine infections. ${ }^{18}$ It is known that some individuals develop T-cell immunity that will not have been detected by the methods used in this report. This will have diminished the size of the effect measured by an unquantifiable amount and as such, the data presented here likely represents the minimum level of protection afforded.

Regular screening of staff was not carried out at the time these data were collected. As such, a systematic assessment of the risk of asymptomatic infection and reinfection is not possible. Similarly, asymptomatic screening on outbreak wards was limited by testing capacity at times and when carried out only occurred at a single point of time. Asymptomatic reinfection is less concerning from an $\mathrm{HCW}$ re-exposure perspective but may have significant implications for infection prevention and control practice. Studies with regular routine screening are required to provide data on this.

The data reported refer to a cohort of HCWs in a high-prevalence setting during a period of shielding. The findings may not be fully generalisable to the wider population, which will include older individuals and those with significant comorbidity.

As reported by case studies, reinfection may occur with phylogenetically distinct variants of SARS-CoV-2. Viral evolution and immunity to coronavirus infections that is not durable mean that vaccination is unlikely to provide life-long protection. This report provides some reassurance that protection will likely last 4 months ( 131 days median) for the majority of individuals. This is in keeping with data from seasonal coronavirus infections, which suggest cycling of infection with different coronavirus strains predominating every 2 to 4 years, ${ }^{19}$ and rechallenge experiments that suggest complete immunity from symptomatic reinfection for at least 1 year if 'reinfected' with the same strain, but only partial immunity when exposed to a heterologous strain. ${ }^{20,21}$ Recent data suggesting durable antibody responses in individuals with asymptomatic and mildly symptomatic infection also support this finding and provide further reassurance. ${ }^{22}$ For three of four immunoassays used, seropositivity rates at 8 months were high $(69.0-91.4 \%){ }^{22}$

To date, the majority of reported reinfections have been milder than the initial infection. ${ }^{4}$ The reinfection case described here was also a milder infection. The initial infection required a 
7-day inpatient admission and supplemental oxygen whereas the reinfection, while symptomatic, did not require any active treatment and did not result in admission. As such, it is hoped that vaccination will provide protection against severe disease even if protection from reinfection is incomplete.

\section{Conclusion}

This report suggests that prior SARS-CoV-2 infection offers significant protection against reinfection. However, evidence of reinfection in our data and other publications support the continued use of infection control and public health pandemic control measures after previous infection and/or vaccination while a more complete picture on the risks of reinfection is established. Duration of protection for at least 4 months for the majority of individuals as evidenced in this data is reassuring both for people who have been previously infected and for the vaccination campaign, although vaccine escape mutants may impact the latter While reinfection is well documented, it is likely that it will become less common with time as repeated opportunity for exposure decreases as a consequence of increased population immunity.

\section{Summary}

\section{What is known?}

$>$ Reinfection with SARS-CoV-2 is possible even after a relatively short interval (months).

$>$ Reinfection with seasonal coronavirus is commonplace.

$>$ Seasonal coronavirus rechallenge experiments suggest complete immunity from symptomatic reinfection for at least 1 year if 'reinfected' with the same strain, but only partial immunity when exposed to a heterologous strain.

$>$ Seasonal coronavirus strain infection is cyclical with certain strains predominating every $2-4$ years.

$>$ Protection from infection with SARS-CoV-2 post vaccination lasts at least 2 months.

$>$ Natural infection with SARS-CoV-2 offers protection from reinfection for an unknown period.

\section{What is the question?}

What is the protective effect of previous COVID-19 infection on reinfection in a high-prevalence hospital setting?

\section{What was found?}

Evidence of previous COVID-19 infection (positive PCR result and/or positive antibody test) in health personnel reduced the attack rate significantly from $24.59 \%$ to $0.87 \%$ (odds ratio $0.027,95 \%$ CI $0.004-0.195, p<0.001$ ) and this protection from reinfection lasted 4 months for the majority of individuals (observation period 99-168 days, median - 131 days).

\section{What is the implication for practice now?}

This report suggests that prior SARS-CoV-2 infection offers significant protection against reinfection. However, evidence of reinfection in our data and other publications support the continued use of infection control and public health pandemic control measures after previous infection and/or vaccination whilst a more complete picture on the risks of reinfection is established. Duration of protection for at least 4 months for the majority of individuals as evidenced in this data is reassuring both for people that have been previously infected and for the vaccination campaign, although vaccine escape mutants may impact the latter.

\section{References}

1 World Health Organization. WHO Coronavirus (COVID-19) Dashboard, 2021. www.covid19.who.int/ [Accessed 17 June 2021]

2 Public Health England. Weekly Coronavirus Disease 2019 (COVID-19) Surveillance Report, 2020. www.assets.publishing.service.gov.uk/ government/uploads/system/uploads/attachment_data/file/ 923668/Weekly_COVID19_Surveillance_Report_week_40.pdf [Accessed 12 December 2020].

3 Polack FP, Thomas S], Kitchin N et al. Safety and efficacy of the BNT162b2 mRNA Covid-19 vaccine. N Engl ] Med 2020;383: 2603-15.

4 Yahav D, Yelin D, Eckerle I et al. Definitions for COVID-19 reinfection, relapse and PCR re-positivity. Clin Microbiol Infect 2021;27:315-18.

5 Larson D, Brodniak SL, Voegtly L] et al. A case of early re-infection with SARS-CoV-2. Clin Infect Dis 2020; ciaa1436.

6 Tillett RL, Sevinsky JR, Hartley PD et al. Genomic evidence for reinfection with SARS-CoV-2: a case study. Lancet Infect Dis 2021;21:52-8.

7 To KK, Hung IF, Ip JD et al. COVID-19 re-infection by a phylogenetically distinct SARS-coronavirus- 2 strain confirmed by whole genome sequencing. Clin Infect Dis 2020; ciaa1275.

8 West J, Everden S, Nikitas N. A case of COVID-19 reinfection in the UK. Clin Med 2021;21:e52-e53.

9 Van-Elslande J, Vermeersch P, Vandervoort K et al. Symptomatic SARS-CoV-2 reinfection by a phylogenetically distinct strain. Clin Infect Dis 2020; ciaa1330.

10 Elecsys Anti-SARS-CoV-2, 2020. www.fda.gov/media/137605/ download [Accessed 12 December 2020].

11 Kohmer N, Westhaus S, Ruehl C, Ciesek S, Rabenau HF. Brief clinical evaluation of six high-throughput SARS-CoV-2 IgG antibody assays. J Clin Virol 2020;129:104480.

12 Mueller L, Ostermann PN, Walker A et al. Sensitivity of commercial Anti-SARS-CoV-2 serological assays in a high-prevalence setting. Eur J Clin Microbioal Infect Dis 2021;40:1063-71.

13 MedCalc Software Ltd. Odds ratio calculator. www.medcalc.org/ calc/odds_ratio.php [Accessed 17 ]une 2021].

14 Sheskin DJ. Handbook of parametric and nomparametric statistical procedures. 3rd edn. Boca Raton: Chapman \& Hall/CRC, 2004:542.

15 Abu-Raddad L], Chemaitelly H, Malek JA et al. Assessment of the risk of SARS-CoV-2 reinfection in an intense re-exposure setting. Clin Infect Dis 2020; ciaa1846.

16 Harvey RA, Rassen JA, Kabelac CA et al. Real-world data suggest antibody positivity to SARS-CoV-2 is associated with a decreased risk of future infection. medRxiv [Preprint] 2020. https://doi.org/ 10.1101/2020.12.18.20248336.

17 Lumley SF, O'Donnell D, Stoesser NE et al. Antibody status and incidence of SARS-CoV-2 infection in health care workers. N Engl ] Med 2021:384:533-40.

18 Watson J, Whiting P, Brush J. Interpreting a Covid-19 test result. BMJ 2020;369:m1808.

19 Monto AS. Medical reviews coronavirus. Yale J of Biol Med 1974;47:234-51.

20 Reed SE. The behaviour of recent isolates of human respiratory coronavirus in vitro and in volunteers: evidence of heterogeneity among 229E-related strains. J Med Virol 1984;13:179-92.

21 Callow KA, Parry HF, Sergeant M, Tyrrell DA. The time course of the immune response to experimental coronavirus infection of man. Epidemiol Infect 1990:105:435-46.

22 Choe PG, Kim K, Kang CK et al. Antibody responses 8 months after asymptomatic or mild SARS-CoV-2 infection. Emerg Infect Dis 2021:27:928-31.

Address for correspondence: Favian Narrainen, Public Health Wales Microbiology, Singleton Hospital, Sketty Lane, Swansea SA2 8QA, UK.

Email: favian@live.co.uk 